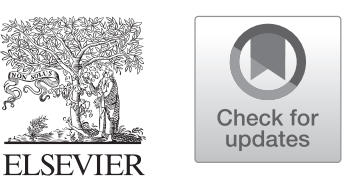

\title{
Primary Prophylaxis for Gastrointestinal Bleeding in Children With Biliary Atresia and Portal Hypertension Candidates for Liver Transplantation: A Single-Center Experience
}

\author{
R. Angelico ${ }^{a, *, \dagger}$, A. Pietrobattista ${ }^{b, \dagger}$, M. Candusso ${ }^{b}$, S. Tomarchio ${ }^{b}$, M. Pellicciaro ${ }^{a}$, D. Liccardo ${ }^{b}$, \\ M.S. Basso ${ }^{b}$, C. Grimaldi ${ }^{a}$, M.C. Saffioti ${ }^{a}$, F. Torroni ${ }^{c}$, L. Dall'Oglio ${ }^{c}$, G. Torre ${ }^{b}$, and M. Spada ${ }^{a}$ \\ aDivision of Abdominal Transplantation and Hepatobiliopancreatic Surgery, Bambino Gesù Children's Research Hospital IRCCS, Rome, \\ Italy; 'Division of Hepatology and Gastroenterology, Bambino Gesù Children's Research Hospital IRCCS, Rome, Italy; and 'Division of \\ Endoscopic and Digestive Surgery, Bambino Gesù Children's Research Hospital IRCCS, Rome, Italy
}

\begin{abstract}
Background. Cirrhosis for biliary atresia (BA) is associated with risk of gastrointestinal bleeding (GB) from gastroesophageal varices due to portal hypertension. Primary prophylaxis of GB is controversial in children who are candidates for liver transplantation (LT). The aim of the study was to define the management of gastroesophageal varices and to identify the benefit of primary prophylaxis for GB in BA children waiting for LT.

Methods. A retrospective single-center study including all BA children listed for LT in 2008-2016. Clinical, endoscopical, and biochemical data were analyzed.

Results. Of 82 children, $50(61 \%)$ did not receive primary prophylaxis and did not present any episode of bleeding, $16(19.5 \%)$ underwent primary prophylaxis, and $16(19.5 \%)$ presented spontaneous GB and received secondary prophylaxis. Children without primary prophylaxis and GB were younger than patients with primary prophylaxis and those with GB (7.7 years [range, $4.1-37.9$ years] vs 11.2 years [range, $5.1-43$ years]; $P=.03$ vs 10.7 years [range, 6.9-39.9 years], respectively; $P=.004$ ). Seventy-five percent of GB occurred in children older than 8 months. Fifteen $(93.8 \%)$ children with GB presented esophageal varices (grade III $=10[62.5 \%]$ ) and $10(62.5 \%)$ required endoscopic treatments, consisting mainly of sclerotherapy. Median time to LT was similar for children with or without bleeding (2 months [range, 0-17.7 months] vs 2.2 months [0-17.9 months], respectively; $P=.89$ ). After 45.5 months (range, 13.7-105.5 months) of follow-up, the overall patient survival was $97.6 \%$. At the intention-to-treat analysis, the survival rate was $100 \%$ for patients without bleeding episode and $87.5 \%$ for children with GB $(P=.16)$.

Conclusions. Despite the risk of GB being not clinically predictable in children with BA waiting for LT, our experience suggests that primary prophylaxis of GB might be unnecessary in children younger than 6 months, while it should be considered in older children. Thus, the occurrence of GB does not delay the timing of transplantation.
\end{abstract}

B ILIARY atresia (BA) is a progressive fibro-obliterative cholangiopathy presenting only in infancy with a prevalence ranging from 1 in 5000 to 18,000 newborns [1]. The hepatoportoenterostomy (HPE), also known as Kasai procedure, provides a means of relieving extrahepatic biliary obstruction to permit bile flow; however, it is not a curative procedure [2,3]. Despite HPE being performed in a timely fashion, advancing fibrosis of the liver is associated with
Grant information: no funding or grant to declare.

*Address correspondence to Roberta Angelico, MD, FEBS, Division of Abdominal Transplantation and Hepatobiliopancreatic Surgery Bambino Gesù, Children's Research Hospital IRCCS, Piazza Sant'Onofrio 4, 00146 Rome, Italy. Tel: 003906 68592862, Fax: 003906 685912863. E-mail: roberta.angelico@ gmail.com

${ }^{\dagger}$ Both authors contributed equally for first authorship

$0041-1345 / 18$ https://doi.org/10.1016/j.transproceed.2018.04.074 
portal hypertension $(\mathrm{PH})$ in a significant number of patients after successful HPE [4]. The development of PH, which is the commonest complication of end-stage liver disease (ESLD), has a significant impact on the pretransplant health and post-transplant outcomes of children with BA and ESLD [5,6]. Gastrointestinal bleeding (GB) from esophageal and gastric varices is the most serious consequence of PH causing significant morbidity $[7,8]$. Therefore, liver transplantation (LT) is ultimately required for BA patients with severe $\mathrm{PH}$ during childhood [9].

For adult cirrhotic patients, gastroesophageal varices screening is recommended during the pre-LT evaluation. The primary prophylaxis of bleeding with either $\beta$-blockers or both esophageal varices band ligation (EVBL) and esophageal sclerotherapy (EST) is routinely performed $[10,11]$. However, there is no consensus for this practice in children because of lack of high-quality evidence favoring prophylactic treatment of $\mathrm{PH}$. Moreover, which patients with BA should undergo screening endoscopy for varices remains unclear.

Herein, we report our experience on BA children with ESLD and PH listed for LT, focusing on the management of gastroesophageal varices in pediatric LT candidates.

\section{MATERIAL AND METHODS Study Design}

A retrospective single-center study was conducted analyzing all children with BA and PH listed for LT between January 2008 and January 2016 at the Bambino Gesù Children Hospital IRCCS of Rome. The aim of this study was to define the management of gastroesophageal varices in BA children who are LT candidates and to identify the risk factors for spontaneous GB.

Demographic characteristics of BA children who are LT candidates included sex, age at the time of inscription on LT waiting list, previous HPE procedure, Pediatric End-Stage Liver Disease (PELD) score, presence of ascites and splenomegaly, reverse portal vein flow at the abdominal ultrasonography scan, and cholangitis episodes (defined as a pyrexia illness not attributed to other sources, treated with intravenous antibiotics). Biochemical data such as blood platelet count, international normalized ratio, serum total bilirubin, and albumin level were also collected at the time of LT evaluation. Occurrence of spontaneous GB, defined as hematemesis with or without associated melena treated with red blood cell transfusion, was recorded. Complications after GB were evaluated as well as GB recurrence, which was considered if episodes of GB reoccurred after commencing endoscopic treatment.

\section{Gastrointestinal Endoscopy Management}

During the observation period at our institution, gastroesophageal varices surveillance by upper gastrointestinal endoscopy was not mandatory for children with $\mathrm{BA}$ and $\mathrm{PH}$ as primary prophylaxis. Thus, upper gastrointestinal endoscopic procedure was performed as primary prophylaxis in selected children older than 6 months according to patient's clinical status. All children who presented a spontaneous GB were treated according to the GB protocol consisting of upper gastrointestinal endoscopy within 72 hours form the bleeding, red blood cell transfusion if hemoglobin was $<8 \mathrm{mg} / \mathrm{dL}$, intravenous antibiotics, and octreotide infusion during the period of active bleeding; after the first bleeding, patients received secondary prophylaxis for varices surveillance.

The endoscopic findings were recorded and graded as follows: (1) number and grade of esophageal varices according to the Paquet classification [12] (grade I: when varices were flattened by insufflation; grade II: when varices were not flattened by insufflation, but were separated by healthy mucosa; grade III: when varices were not flattened by insufflation and were confluent and obstructing the lumen); (2) presence of red wale markings on the esophageal mucosa [13]; (3) presence of gastric varices along the cardia (gastroesophageal varices 1 and gastroesophageal varices 2) [14]; (4) presence of portal hypertensive gastropathy. As per our center's protocol, patients presenting grade III varices at the endoscopy received endoscopic intervention. The endoscopic treatment of esophageal varices included EVBL or EST. Gastrointestinal endoscopy was performed under general anesthesia or midazolam sedation $(0.2-0.3 \mathrm{mg} / \mathrm{kg})$. Each endoscopy was performed or supervised by 2 senior pediatric endoscopists, and the criteria of recording the endoscopic findings did not change throughout the study period. Informed consent of the parents was obtained before each endoscopic procedure.

\section{Statistical Analysis}

Data were collected from a prospectively consecutive database (Microsoft Access 2.0; Microsoft Corporation, Redmond, Wash, United States). Patient characteristics and clinical data are shown (wherever applicable) as either median (range) or mean (SD). Univariate data were analyzed using the Mann-Whitney test and Fisher exact test. A $P$ value $<.05$ was considered significant. Normal distribution continuous data were analyzed by parametric test ( $t$ test). Survival rate and bleeding-free survival were analyzed using the Kaplan-Meier method. For overall survival the start of follow-up was the date of listing for LT and the end of follow-up was the date of death or of the last information. For bleedingfree survival, the start of follow-up was the date of the listing for LT and the end of follow-up was the date of spontaneous GB episode. Cox regression was used for the multivariate analysis. The program used for statistical analysis was SPSS 13.0 (Chicago, Ill, United States) for Windows.

\section{RESULTS}

\section{Study Population}

Of 155 children listed for LT at our institution during the study period, $82(52.9 \%)$ patients (48 [58.5\%] female, median age 8.6 months [range, 4.1-43 months]) were affected by BA and PH and were enrolled in the study. Demographic and clinical characteristics of the study population at the time of listing for LT are summarized in Table 1.

During the observational period, $50(61 \%)$ children did not receive primary prophylaxis for GB and did not present any episode of GB (group 1), 16 (19.5\%) children underwent primary prophylaxis for GB (group 2), and 16 (19.5\%) children presented spontaneous GB and underwent upper gastrointestinal endoscopy after the bleeding episode (group 3) (Fig 1).

Patient characteristics of the 3 groups at the time of LT listing are detailed in Table 2. Children who did not receive primary prophylaxis for GB were significantly younger (group 1: 7.7 years [range, 4.1-37.9 years]) than patients 
Table 1. Overall Characteristics of Children With Biliary Atresia and Portal Hypertension at the Time of Listing for Liver Transplantation

\begin{tabular}{lc}
\hline \multicolumn{1}{c}{ Variables } & Values \\
\hline No. of patients & 82 \\
Age (range), mo & $8.6(4.1-43)$ \\
Weight (range), kg & $7.3(4.4-21)$ \\
Sex, No. (female, \%) & $48(58.5)$ \\
Portoenterostomy procedure, No. (\%) & $60(73.2)$ \\
Splenomegaly, No. (\%) & $80(97.6)$ \\
Reversed portal flow at US, No. (\%) & $32(39)$ \\
Cholangitis episode, No. (\%) & $22(26.8)$ \\
Ascites, No. (\%) & $60(73.2)$ \\
PELD score (range) & $22(1-45)$ \\
Total bilirubin (range), mg/dL & $13.5(0.8-34)$ \\
Platelets (range), $\times 10^{3} / L$ & $179(18-654)$ \\
Albumin (range), g/dL & $3.1(2.3-4.2)$ \\
INR (range) & $1.36(0.9-2.9)$ \\
Endoscopy for primary prophylaxis after & $16(59)$ \\
listing, No. (\%) & \\
\hline
\end{tabular}

Abbreviations: INR, international normalized ratio; LT, liver transplant; PELD, Pediatric End-Stage Liver Disease; US, ultrasonography.

who underwent primary prophylaxis (group 2: 11.2 years [range, 5.1-43 years]; $P=.03$ ) and those who presented a spontaneous GB (group 3: 10.7 years [range, 6.9-39.9 years]; $P=.004)$. Moreover, children without primary prophylaxis and GB episode had lower body weight at the time of listing for LT ( $7 \mathrm{~kg}$ [range, $4.5-17 \mathrm{~kg}$ ] in group $1 \mathrm{vs}$ $10 \mathrm{~kg}$ [range, $6.1-20 \mathrm{~kg}$ ] in group $2[P<.001] \mathrm{vs} 8.9 \mathrm{~kg}$ [range, $4.7-30 \mathrm{~kg}]$ in group $3[P=.03])$. No difference was found between the 3 groups in terms of sex, previous HPE procedure, episodes of bacterial cholangitis, PELD score, presence of ascites, splenomegaly, and reversed portal flow on the Doppler ultrasonography, while biochemical tests showed lower level of serum total bilirubin and international normalized ratio in children who experienced GB (group 3).

\section{Clinical Outcomes and Endoscopic Management}

During the study period, $16(19.5 \%)$ children listed for LT experienced spontaneous $\mathrm{GB}$ at a median age of 12.5 months (range, 7.4-43 months). Of note, 12 (75\%)

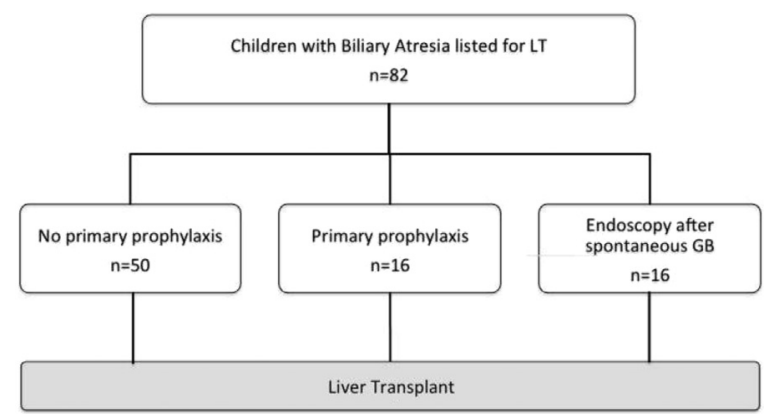

Fig 1. Study population. Abbreviations: GB, gastrointestinal bleeding; LT, liver transplantation. patients presented a spontaneous GB after 8 months of age, and only $1(6.3 \%)$ patient did so before 6 months of age (Fig 2).

After the bleeding episode, all patients were admitted to the hospital and received medical treatment consisting of red blood cell transfusion if hemoglobin was $<8 \mathrm{~g} / \mathrm{dL}$, octreotide infusion, and intravenous antibiotic; when hemodynamic stabilization was ensured, all children underwent upper gastrointestinal endoscopy within 3 days (range, 1-5 days) from the bleeding episode. All patients recovered from the bleeding episode; 7 (43.8\%) children with spontaneous GB required intensive therapy unit admission.

At the first endoscopy performed after the GB episode, esophageal varices were found in $15(93.8 \%)$ patients, of which grade III esophageal varices were observed in 10 $(62.5 \%)$ cases, red wale markings in $8(50 \%)$ cases, and signs of active bleeding in $4(25 \%)$ cases (Table 3$)$. Endoscopic treatment was performed in $10(62.5 \%)$ patients, consisting of sclerotherapy and banding in $8(50 \%)$ and $2(12.5 \%)$ cases, respectively. Portal hypertensive gastropathy was observed in $13(81.3 \%)$ patients, and gastric varices along the cardia in was observed in $5(31.3 \%)$ children.

After the first endoscopy, all children underwent secondary prophylaxis with a median number of 2 endoscopic sessions (range, 1-5 sessions) for each patient before LT. Four $(25 \%)$ children presented a second spontaneous bleeding before the next protocol endoscopy: at the subsequent endoscopy, all of them showed portal hypertensive gastropathy, associated with grade III varices and red wale markings in 2 cases (treated by sclerotherapy) and gastric varices along the cardia in 2 cases; all patients recovered without major sequel from the second bleeding episode.

Sixteen $(19.5 \%)$ children listed for LT received upper gastrointestinal endoscopy as primary prophylaxis at a median age of 14.3 months (range, $7-43$ months). In this group, esophageal varices were found in $8(50 \%)$ cases (Table 3); grade III varices and red wale markings were observed in $3(18.8 \%)$ patients, who were treated by sclerotherapy $(\mathrm{n}=2[12.5 \%])$ and banding $(\mathrm{n}=1[6.3 \%])$. The median number of endoscopies for primary prophylaxis was 1 (range, 1-6). No complications were observed in children who underwent endoscopy for primary prophylaxis. Only 1 patient who started the primary prophylactic protocol for GB presented a spontaneous bleeding before the next session of endoscopy: at the endoscopy, esophageal varices grade III with red wale marking were found and treated by sclerotherapy, with subsequent recovery of the patient from the bleeding episode.

\section{Patient Survival and Intention-to-Treat Analysis}

After a median follow-up of 45.5 months (range, 13.7-105.5 months), the overall patient survival was $97.6 \%$. Except 1 patient who died while on the waiting list, all children $(98.8 \%)$ received LT within a median time of 2.1 months (range, 0-17.9 months) from LT listing. The median time 
Table 2. Patient Characteristics Classified on Gastrointestinal Bleeding Prophylaxis at Listing for Liver Transplantation.

\begin{tabular}{|c|c|c|c|c|c|c|}
\hline Variables & $\begin{array}{l}\text { No Primary } \\
\text { Prophylaxis } \\
\text { (Group 1) }\end{array}$ & $\begin{array}{l}\text { Primary Prophylaxis } \\
\text { (Group 2) }\end{array}$ & $\begin{array}{c}\text { Spontaneous GB, } \\
\text { Secondary Prophylaxis } \\
\text { (Group 3) }\end{array}$ & $\begin{array}{l}P \text { Value } \\
1 \text { vs } 2\end{array}$ & $\begin{array}{l}P \text { Value } \\
1 \text { vs } 3\end{array}$ & $\begin{array}{c}P \text { Value } \\
2 \text { vs } 3\end{array}$ \\
\hline No. of patients & 50 & 16 & 16 & - & - & - \\
\hline Age (range), mo & $7.7(4.1-37.9)$ & $11.2(6.1-43)$ & $10.7(6.9-39.9)$ & $.03^{*}$ & $.004^{*}$ & .62 \\
\hline Weight (range), kg & $7(4.5-17)$ & $10(6.1-20)$ & $8.9(8-30)$ & $<.001^{*}$ & $.03^{*}$ & .63 \\
\hline Sex, No. (female, \%) & $29(58)$ & $7(43.8)$ & $12(75)$ & .39 & .25 & .15 \\
\hline Portoenterostomy procedure, No. (\%) & $36(72)$ & $11(68.8)$ & $13(81)$ & $>.99$ & .48 & .15 \\
\hline Splenomegaly, No. (\%) & $49(98)$ & $15(93.8)$ & $16(100)$ & .43 & $>.99$ & $>.99$ \\
\hline Reversed portal flow at US, No. (\%) & $17(34)$ & $6(37.5)$ & $9(56.3)$ & $>.99$ & .14 & .48 \\
\hline Cholangitis episode, No. (\%) & $12(24)$ & 5 (31.3) & 5 (31.3) & .75 & .74 & $>.99$ \\
\hline Ascites, No. (\%) & $38(76)$ & $12(75)$ & $10(62.5)$ & $>.99$ & .34 & .70 \\
\hline PELD score (range) & $23(1-45)$ & $20(6-38)$ & $17(2-43)$ & .14 & .12 & .83 \\
\hline Total bilirubin (range), $\mathrm{mg} / \mathrm{dL}$ & $14.6(4.1-34)$ & $14.1(5.1-25)$ & $8.8(0.8-22.5)$ & .43 & $<.001^{*}$ & $.02^{*}$ \\
\hline Platelets (range), $\times 10^{3} / \mathrm{L}$ & $189(18-546)$ & $126(65-654)$ & $175(69-488)$ & .24 & .68 & .58 \\
\hline Albumin (range), $\mathrm{g} / \mathrm{dL}$ & $3.1(2.3-4)$ & $3.2(2.4-3.5)$ & $3.3(2.6-4.2)$ & .84 & $.02^{*}$ & .09 \\
\hline INR (range) & $1.4(1-2.9)$ & $1.3(1-2.8)$ & $1.2(0.9-1.8)$ & .69 & .12 & .10 \\
\hline Time from listing to LT (range), mo & $1.8(0-17.9)$ & $5.3(0.1-14.4)$ & $2(0.1-17.7)$ & $.03^{*}$ & .17 & .84 \\
\hline
\end{tabular}

Characteristics of children with biliary atresia and portal hypertension classified on gastrointestinal bleeding prophylaxis.

Abbreviations: GB, gastrointestinal bleeding; INR, international normalized ratio; LT, liver transplantation; PELD, Pediatric End-Stage Liver Disease; US, ultrasonography.

${ }^{\star} P<.05$.

from listing to LT was similar for children who experienced a spontaneous GB and for those who did not bleed (2 months [range, 0-17.7 months] vs 2.2 months [range, 0-17.9 months]; $P=.89$ ) (Fig 3).

The intention-to-treat analysis showed that the patient survival from the time of listing for LT to the last follow-up

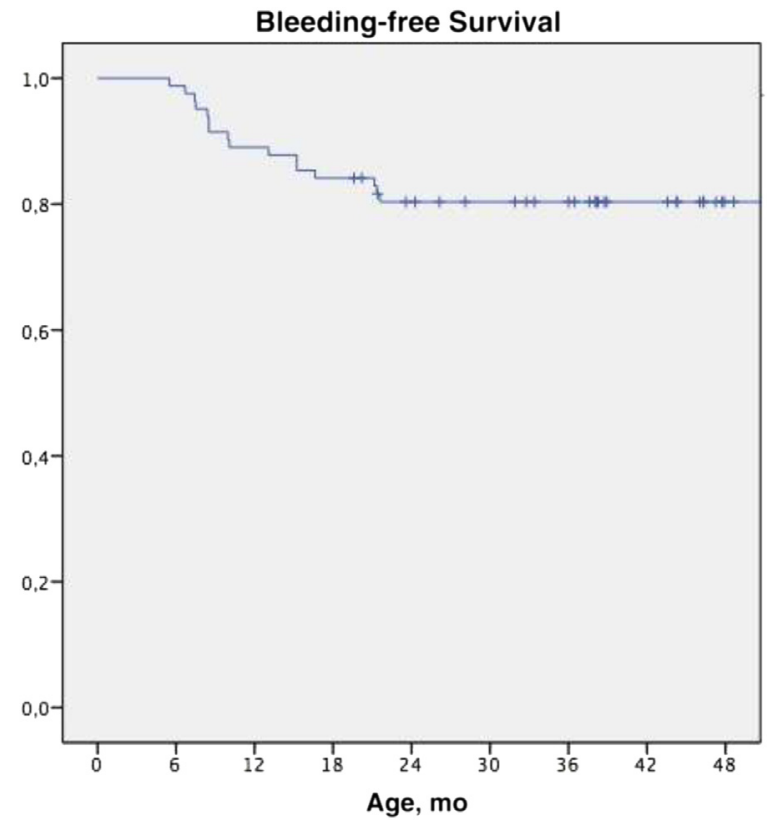

Fig 2. Bleeding-free survival. Kaplan-Meier survival estimates the bleeding-free survival in children with biliary atresia and portal hypertension. More than $50 \%$ of children presented at least 1 episode of spontaneous bleeding after 12 months of age. Children who underwent primary prophylaxis were excluded from the Kaplan-Meier survival. was $100 \%$ for patients who did not experience spontaneous GB and did not have primary prophylaxis as well as for those who underwent primary prophylaxis and $87.5 \%$ for children who presented a spontaneous GB $(P=.16)$ (Fig 4). During the observational period, 1 patient died of multiorgan failure caused by bacterial sepsis while waiting for LT

Table 3. Endoscopic Findings Recorded in Children With Biliary Atresia And Portal Hypertension.

\begin{tabular}{lccc}
\hline \multicolumn{1}{c}{ Variables } & $\begin{array}{c}\text { Endoscopy } \\
\text { for GB }\end{array}$ & $\begin{array}{c}\text { Endoscopy } \\
\text { for Primary } \\
\text { Prophylaxis }\end{array}$ & $\begin{array}{c}P \\
\text { Value }\end{array}$ \\
\hline No. of patients & 16 & 16 & - \\
Endoscopic pattern, No. (\%) & & & \\
$\quad$-Esophageal varices & $15(93.8)$ & $8(50)$ & $.02^{*}$ \\
$\quad$ Grade I & $1(6.3)$ & $4(25)$ & .33 \\
$\quad$ Grade II & $4(25)$ & $1(6.3)$ & .33 \\
$\quad$ Grade III & $10(62.5)$ & $3(18.8)$ & $.03^{*}$ \\
-Esophageal red wale & $8(50)$ & $3(18.8)$ & .14 \\
$\quad$ markings & & & \\
-Gastric varices along the & $5(31.3)$ & $3(18.8)$ & .69 \\
$\quad$ cardia & $13(81.3)$ & $10(62.5)$ & .15 \\
-Portal hypertensive & & & \\
$\quad$ gastropathy & $10(62.5)$ & $3(18.8)$ & $.03^{*}$ \\
Endoscopic treatment, No. (\%) & $8(50)$ & $2(12.5)$ & $.02^{*}$ \\
-Sclerotherapy & $2(12.5)$ & $1(6.3)$ & .60 \\
$\quad$-Banding & $2(1-5)$ & $1(1-6)$ & .17 \\
No. of endoscopic sessions & $4(25)$ & $1(6.3)$ & .33 \\
Bleeding before the next & & & \\
$\quad$ endoscopic session, No. (\%) & & & \\
Bleeding requiring ITU & $7(43.8)$ & $0(0)$ & $.007^{*}$ \\
admission, No. (\%) & & & \\
\hline
\end{tabular}

Data refers to endoscopic findings observed in children with biliary atresia and portal hypertension at the first endoscopy performed after spontaneous gastrointestinal bleeding and in children who underwent endoscopy as primary prophylaxis.

Abbreviations: GB, gastrointestinal bleeding; ITU, intensive therapy unit; $N$, number; LT, liver transplant.

${ }^{\star} P<.05$. 


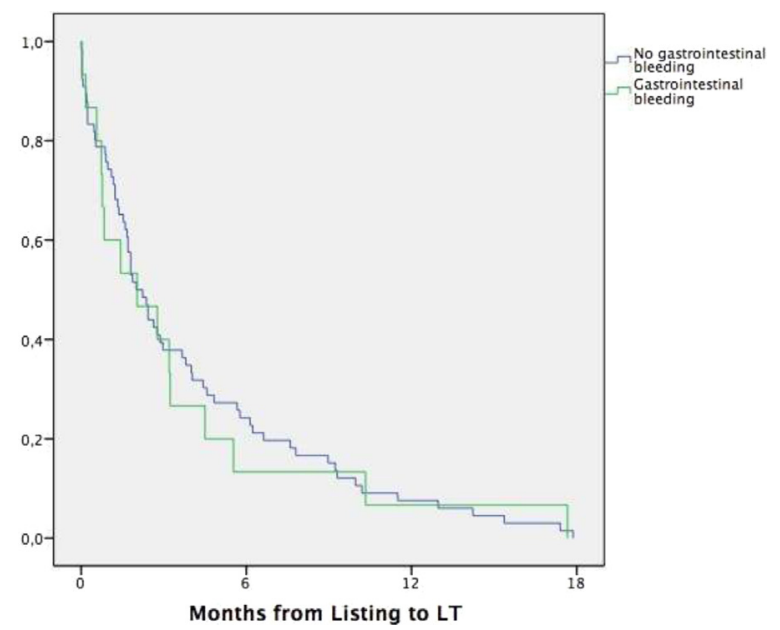

Fig 3. Time from listing to liver transplantation. The median time from the inscription on the LT waiting list and the time of transplant in children with biliary atresia and portal hypertension with or without spontaneous gastrointestinal bleeding. Abbreviation: LT, liver transplantation.

after 12 days from spontaneous GB at the age of 9 months. One 7-month-old infant with no previous HPE underwent urgent LT after $1 \mathrm{~GB}$ episode and died of medical and surgical complications related to multidrug bacterial infection 3 months after LT.

The univariate and multivariate analysis did not identify any statistically significant factors predictive of GB in children with BA and $\mathrm{PH}$.

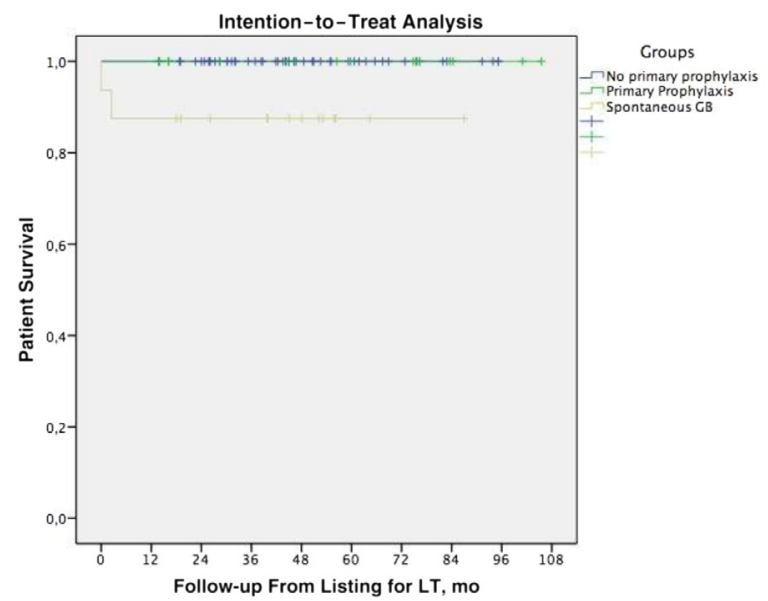

Fig 4. Intention-to-treat analysis for children with biliary atresia and portal hypertension. Kaplan-Meier survival estimates the overall probability of patient survival in children with biliary atresia and portal hypertension from the time of listing for liver transplant to the last follow-up. Children were classified according to gastrointestinal bleeding occurrence. Abbreviations: GB, gastrointestinal bleeding; LT, liver transplantation.

\section{DISCUSSION}

LT had a profound impact on the health care evolution of children with ESLD, and it represents the most effective treatment for many patients with chronic liver failure from a variety of causes. BA is the most common indication for LT in children, representing more than $50 \%$ of cases in most series. The usual investigations for potential LT recipients include a wide range of medical, social, laboratory, and radiologic investigations. The aim of this process is to enable staging of the primary diagnosis, the assessment of comorbidities, and the identification of technical and/or surgical challenges for LT in order to optimize the overall outcomes and avoid potential complications [15-19]. The management of $\mathrm{PH}$ in children with $\mathrm{BA}$ waiting for LT represents a crucial point for pediatric hepatologists and transplant surgeons.

Many adult studies highlight advantages in performing upper gastrointestinal endoscopy in the pre-LT assessment as relevant gastroesophageal varices pathology might impact the patient selection and management before LT [16-18]. In a series of adult LT candidates, the rate of gastroesophageal varices was up to $64 \%$ at the pre-LT endoscopy; however, banding ligation was realized in only $7.6 \%$ of patients and $22 \%$ were started on $\beta$-blocker treatment [16].

In children, the management of $\mathrm{PH}$ remains poorly studied, and the use of $\beta$-blocker therapy for primary prophylaxis of variceal hemorrhage is controversial and not convincingly effective [10]. In childhood, the mortality of a first esophageal variceal bleeding is extremely low and the morbidity is not well known [10]. Therefore, many pediatric hepatology centers favor endoscopic secondary prophylaxis of child with variceal hemorrhage over primary prophylaxis, which brings more debate.

Unlike in adults, in children with BA and PH who are LT candidates, there is a lack of data on the benefit and efficacy of upper GB prophylaxis because of the absence of randomized controlled trials and the difficulty of the endoscopic procedure in very small children $(<10 \mathrm{~kg})$, particularly for EVBL. To date, only 1 published study focused on the endoscopic evaluation of esophageal varices in children with ESLD under LT evaluation [19].

Our study was based on very small BA children with advanced ESLD and an overall clear indication for LT due to the presence of liver decompensation at the time of evaluation (median PELD score, 21 [range, 1-45]). During the study period, we observed that $20.5 \%$ of children had at least 1 episode of GB after listing for LT. Children presenting spontaneous GB were older than those without GB episode, which was expected considering the progressive course of ESLD. Moreover, $75 \%$ of children presented a gastrointestinal bleeding after 8 months of life, while only $2 \%$ did so before 6 months of age.

The laboratory and clinical characteristics at the time of listing for LT were similar for patients with and without GB, except for the serum bilirubin level, which was lower in the bleeding group and could be related to the progressive 
worsening of $\mathrm{PH}$ in BA patients with good or partial bile drainage after HPE. Compared with the recent study of $\mathrm{Ng}$ et al [19], we did not find any difference of the prothrombin time and the association between severity of synthetic dysfunction and PH. Furthermore, the PELD score, as risk indicator of severity and mortality for liver disease, was slightly lower in children who presented a spontaneous bleeding compared with those who did not.

To avoid unnecessary endoscopy, especially in low-risk patients, many studies have been carried out to identify noninvasive factors that may predict the presence of gastroesophageal varices [20-22]. Thus, the number of noninvasive tools for gastroesophageal varices has been growing rapidly over the years, including biochemical, clinical, and ultrasonography parameters [23,24]. However, in our series we did not find difference in variables such as platelet count, spleen measure, and reversal flow in portal vein.

In the current report, 39\% of children underwent gastrointestinal endoscopy, which were event-driven in 50\% of cases. Children undergoing endoscopy for primary prophylaxis and after spontaneous GB showed similar demographic characteristics; however, the incidence of esophageal varices and the need for treatment were significantly different. In our series, esophageal varices were present in $87.5 \%$ of patients who underwent upper gastrointestinal endoscopy for spontaneous GB and in 50\% of children who underwent endoscopy for primary prophylaxis. The endoscopic treatment was performed in $62.5 \%$ of patients who had event-driven endoscopy compared with only $19 \%$ of children in the primary prophylaxis group. In our center the 2 main endoscopic therapeutic interventions for varices consisted of EVBL or EST. Although EST provides an effective treatment for esophageal varices [25,26], it has been associated with possible complications including rebleeding at a rate of $20 \%$ to $30 \%$ and sclerosant-induced esophageal injury that may cause ulceration, bleeding, perforation, or strictures $[27,28]$. On the other hand, EVBL is superior to EST both in terms of efficacy and safety, especially in the pediatric population [27]; however, its feasibility is limited in small children (less than $10-15 \mathrm{~kg}$ or less than 3 years of age) because the banding devices positioned on endoscopes could be too large to pass through the esophagus of a child of this size [29].

In the last years, several authors focused on the role of routine endoscopic surveillance for esophageal varices and on the benefit of prophylactic sclerotherapy in very small children with BA in order to reduce the risk of GB and achieve esophageal varices eradication before LT [25,30,31], which represents a crucial factor for BA children with severe $\mathrm{PH}$ because the intraoperative upper $\mathrm{GB}$ occurrence during the anhepatic phase could be a dramatic event with high risk of mortality.

Despite a recent report showing that pre-emptive endoscopic treatment seems to be effective and well tolerated in young children and that it could help delay LT [25,30,31], clear evidence of the benefit of performing a primary prophylaxis in small BA children has not been demonstrated yet. In our experience, we did not perform primary prophylaxis in children younger than 6 months; except for 1 child, they did not experience GB before LT and had an uneventful outcome after transplant. The choice not to perform primary prophylaxis in very small children was driven mainly by the fact that the endoscopic treatment is often very difficult because of the low body weight; moreover, in very small children the median time to LT is generally limited, as confirmed in our results. In this specific setting of very small candidates for LT ( $<6$ months), who often are clinically poor and fragile because of long-term hospitalization for deep coagulopathy, refractory ascites, or severe infections, we still miss clear evidence supporting primary prophylactic therapy. A full eradication protocol might be difficult to achieve and even more difficult to maintain; thus, it is questionable whether endoscopic surveillance in very small candidates is mandatory pre-LT because the transplant represents the only radical treatment toward both ESLD and its related complications. As stated in previous studies, we need to weigh the risks and benefits of multiple procedures in a nonbleeding child who never bled, when varices have a high chance of recurring, and transplant is sometimes imminent [32,33]. In 2014, the American Association for the study of Liver Disease, the American Society of Transplantation, and the North American Society for Pediatric Gastroenterology, Hepatology and Nutrition published their joint guidelines for the evaluation of pediatric patients for LT for which screening endoscopy for gastroesophageal varices is not recommended [15].

Recently, $\mathrm{Ng}$ et al published the only report on the relevance of endoscopy in children with $\mathrm{PH}$ secondary to ESLD awaiting LT [19]. However, their results failed to provide definitive conclusions on the role of prophylactic pre-LT endoscopy and how it could improve outcome in terms of prevention of bleeding pre-LT. In our analysis we were able to show that the overall survival was similar for patients with and without primary endoscopic prophylaxis and also for patients who underwent endoscopy for spontaneous GB bleeding. Thus, 2 patients with GB died, 1 prior to LT and the other after LT; in both cases, the fatal outcome was caused by complications related to severe nosocomial multidrug resistant bacteria infection rather than as the direct consequence of GB. However, 1 patient acquired multidrug-resistant bacteria during his hospital admission for bleeding management.

Our data confirmed that in BA children the overall death after the first variceal bleeding is infrequent, which has been estimated up to $2 \%$ to $5 \%$ in literature $[34,35]$. These data should be taken into consideration for the decision to perform a surveillance and eradication for esophageal varices vs an LT at an earlier stage. In our series we observed that children experiencing spontaneous GB underwent LT after a similar median time as those who did not experience GB, without any delaying associated with the bleeding event. In our center we perform 
secondary endoscopic prophylaxis after a first variceal bleed, with prompt listing for LT for those children in whom hepatic decompensation is present (failed Kasai procedure).

This study has several limitations, mainly consisting of its retrospective nature, the lack of systematic endoscopic primary prophylaxis for $\mathrm{GB}$, and the low numbers of patients.

In conclusion, our experience suggests that primary prophylaxis of GB might be unnecessary in very small ( $<6$ months) candidates for LT, while it should be considered in older children, because GB is a dramatic event for such small and fragile patients. The occurrence of GB in children awaiting LT does not seems to delay the timing of transplant if children are under close surveillance by the transplant center, which is needed to ensure good outcomes. Our results emphasize the need for future research in pediatric liver disease, ideally randomized controlled trials or multicenter prospective studies able to collect large data in order to determine whether the risk/ benefit ratio truly favors pre-LT endoscopic primary prophylaxis in small BA children who have never experienced a variceal hemorrhage.

\section{REFERENCES}

[1] Chardot C. Biliary atresia. Orphanet J Rare Dis 2006;1:28.

[2] Sokol RJ, Mack C, Narkewicz MR, Karrer FM. Pathogenesis and outcome of biliary atresia: current concepts. J Pediatr Gastroenterol Nutr 2003;37:4-21.

[3] Davenport M. Biliary atresia: clinical aspects. Semin Pediatr Surg 2012;21:175-84.

[4] Houwen RH, Zwierstra RP, Severijnen RS, Bouquet J, Madern G, Vos A, et al. Prognosis of extrahepatic biliary atresia. Arch Dis Child 1989;64:214-8.

[5] Alonso EM, Hackworth C, Whitington PF. Portal hypertension in children. Clin Liver Dis 1997;1:201-22.

[6] Tabasco-Minguillan J, Jain A, Naik M, Weber KM, Irish W, Fung JJ, et al. Gastrointestinal bleeding after liver transplantation. Transplantation 1997;63:60-7.

[7] D'Antiga L. Medical management of esophageal varices and portal hypertension in children. Semin Pediatr Surg 2012;21:211-8.

[8] van Heurn LW, Saing H, Tam PK. Portoenterostomy for biliary atresia: long-term survival and prognosis after esophageal variceal bleeding. J Pediatr Surg 2004;39:6-9.

[9] Shneider BL, Mazariegos GV. Biliary atresia: a transplant perspective. Liver Transpl 2007;13:1482-95.

[10] de Franchis R, Baveno VI Faculty. Expanding consensus in portal hypertension: Report of the Baveno VI Consensus Workshop: stratifying risk and individualizing care for portal hypertension. J Hepatol 2015;63:743-52.

[11] Triantos CK, Burroughs AK. Prevention of the development of varices and first portal hypertensive bleeding episode. Best Pract Res ClinGastroenterol 2007;21:31-42.

[12] Paquet KJ. Prophylactic endoscopic sclerosing treatment of the esophageal wall in varices-a prospective controlled randomized trial. Endoscopy 1982;14:4-5.

[13] Beppu K, Inokuchi K, Koyanagi N, Nakayama S, Sakata H, Kitano S, et al. Prediction of variceal hemorrhage by esophageal endoscopy. Gastrointest Endosc 1981;27:213-8.

[14] Sarin SK, Lahoti D, Saxena SP, Murthy NS, Makwana UK. Prevalence, classification and natural history of gastric varices: a long-term follow-up study in 568 portal hypertension patients. Hepatology 1992;16:1343-9.
[15] Squires RH, Ng V, Romero R, Ekong U, Hardikar W, Emre S, et al. Evaluation of the pediatric patient for liver transplantation: 2014 practice guideline by the American Association for the Study of Liver Diseases, American Society of Transplantation and the North American Society for Pediatric Gastroenterology, Hepatology and Nutrition. Hepatology 2014;60: 362-98.

[16] Weller DA, DeGuide JJ, Riegler JL. Utility of endoscopic evaluations in liver transplant candidates. Am J Gastroenterol 1998;93:1346-50.

[17] Zaman A, Hapke R, Flora K, Rosen H, Benner K. Prevalence of upper and lower gastrointestinal tract findings in liver transplant candidates undergoing screening endoscopic evaluation. Am J Gastroenterol 1999;94:895-9.

[18] Horsley-Silva JL, Vargas HE. Gastrointestinal endoscopy in the cirrhotic patient. Expert Rev Gastroenterol Hepatol 2015;9: 1005-13.

[19] Ng NBH, Karthik SV, Aw MM, Quak SH. Endoscopic evaluation in children with end-stage liver disease associated portal hypertension awaiting liver transplant. J Pediatr Gastroenterol Nutr 2016;63:365-9.

[20] Kim BK, Han KH, Park JY, Ahn SH, Kim JK, Paik YH, et al. A liver stiffness measurement-based, noninvasive prediction model for high-risk esophageal varices in B-viral liver cirrhosis. Am J Gastroenterol 2010;105:1382-90.

[21] Sebastiani G, Tempesta D, Fattovich G, Castera L, Halfon P, Bourliere M, et al. Prediction of oesophageal varices in hepatic cirrhosis by simple serum non-invasive markers: results of a multicenter, large-scale study. J Hepatol 2010;53:630-8.

[22] Ying L, Lin X, Xie ZL, Hu YP, Shi KQ. Performance of platelet count/spleen diameter ratio for diagnosis of esophageal varices in cirrhosis: a meta-analysis. Dig Dis Sci 2012;57: 1672-81.

[23] Alcantara RV, Yamada RM, De Tommaso AM, BellomoBrandão MA, Hessel G. Non-invasive predictors of esophageous varices in children and adolescents with chronic liver disease or extrahepatic portal venous obstruction. J Pediatr (Rio J) 2012;88: $341-6$.

[24] Adami MR, Kieling CO, Schwengber FP, Hirakata VN, Vieira SMG. Noninvasive methods of predicting large esophageal varices in children with intrahepatic portal hypertension. J Pediatr Gastroenterol Nutr 2018;66:442-6.

[25] Duche M, Habes D, Roulleau P, Haas V, Jacquemin E, Bernard O. Prophylactic endoscopic sclerotherapy of large esophagogastric varices in infants with biliary atresia. Gastrointest Endosc $2008 ; 67: 732-7$.

[26] Goncalves ME, Cardoso SR, Maksoud JG. Prophylactic sclerotherapy in children with esophageal varices: long-term results of a controlled prospective randomized trial. J Pediatr Surg 2000;35:401-5.

[27] Zargar SA, Javid G, Khan BA, Yattoo GN, Shah AH, Gulzar GM, et al. Endoscopic ligation compared with sclerotherapy for bleeding esophageal varices in children with extrahepatic portal venous obstruction. Hepatology 2002;36:666-72.

[28] Poza Cordon J, Froilan Torres C, Burgos García A, Gea Rodriguez F, Suárez de Parga JM. Endoscopic management of esophageal varices. World J Gastrointest Endosc 2012;4: $312-22$.

[29] Barth BA, Banerjee S, Bhat YM, Desilets DJ, Gottlieb KT, Maple JT, et al. Equipment for pediatric endoscopy. Gastrointest Endosc 2012;76:8-17.

[30] Lampela H, Kosola S, Koivusalo A, Lauronen J, Jalanko H, Rintala R, et al. Endoscopic surveillance and primary prophylaxis sclerotherapy of esophageal varices in biliary atresia. J Pediatr Gastroenterol Nutr 2012;55:574-9.

[31] Duche M, Ducot B, Ackermann O, Jacquemin E, Bernard O. Progression to high-risk gastroesophageal varices in children with biliary atresia with low-risk signs at first endoscopy. J Pediatr Gastroenterol Nutr 2015;60:664-8. 
[32] Shneider BL, Abel B, Haber B, Karpen SJ, Magee JG, Romero R, et al. Portal hypertension in children and young adults with biliary atresia. J Pediatr Gastroenterol Nutr 2012;55:567-73.

[33] Shneider BL, Brown MB, Haber B, Whitington PF, Schwarz K, Squires R, et al. A multicenter study of the outcome of biliary atresia in the United States, 1997 to 2000. J Pediatr 2006;148:467-74.
[34] Molleston JP, Shneider BL. Preventing variceal bleeding in infants and children: is less more? Gastroenterology 2013;145: 719-22.

[35] Wanty C, Helleputte T, Smets F, Sokal EM, Stephenne X. Assessment of risk of bleeding from esophageal varices during management of biliary atresia in children. J Pediatr Gastroenterol Nutr 2013;56:537-43. 